\title{
Structural and Functional Analysis of Nonheme Iron Enzymes BCMO-1 and BCMO-2 from Caenorhabditis elegans
}

\author{
Weimin Pan ${ }^{1}$, Yong-Ling Zhou ${ }^{1}$, Jian Wang ${ }^{1}$, Huai-En Dai ${ }^{1}$, Xiao Wang ${ }^{1,2}$ and Lin Liu ${ }^{1,2 *}$ \\ ${ }^{1}$ School of Life Sciences, Anhui University, Hefei, China, ${ }^{2}$ Anhui Key Laboratory of Modern Biomanufacturing, Anhui University, \\ Hefei, China
}

Carotenoid metabolism is critical for diverse physiological processes. The nematode Caenorhabditis elegans has two genes that are annotated as $\beta$-carotene 15,15' monooxygenase $(\mathrm{BCMO})$ and are 17 centimorgan apart on chromosome $\mathrm{Il}$, but the function of $\mathrm{BCMO}-1$ and $\mathrm{BCMO}-2$ remains uncharacterized. Sequence homology indicates that the two enzymes belong to the carotenoid cleavage dioxygenase family

OPEN ACCESS

Edited by: Qi Zhang,

Fudan University, China

Reviewed by: Sam P. De Visser, The University of Manchester, United Kingdom Philip Kiser, VA Long Beach Healthcare System, United States

Johannes Von Lintig, Case Western Reserve University, United States

*Correspondence: Lin Liu liulin@ahu.edu.cn

Specialty section:

This article was submitted to Protein Biochemistry for Basic and Applied Sciences,

a section of the journal

Frontiers in Molecular Biosciences

Received: 28 December 2021

Accepted: 17 January 2022

Published: 10 February 2022

Citation:

Pan W, Zhou Y-L, Wang J, Dai H-E, Wang $X$ and Liu L (2022) Structural and Functional Analysis of Nonheme Iron Enzymes BCMO-1 and BCMO-2 from

Caenorhabditis elegans.

Front. Mol. Biosci. 9:844453.

doi: 10.3389/fmolb.2022.844453 that share a seven-bladed $\beta$-propeller fold with a nonheme iron center. Here we determined crystal structures of $\mathrm{BCMO}-1$ and $\mathrm{BCMO}-2$ at resolutions of 1.8 and $1.9 \AA$, respectively. Structural analysis reveals that $\mathrm{BCMO}-1$ and $\mathrm{BCMO}-2$ are strikingly similar to each other. We also characterized their $\beta$-carotene cleavage activity, but the results suggest that they may not act as $\beta$-carotene $15,15^{\prime}$-oxygenases.

Keywords: carotenoid, dioxygenase, $\beta$-propeller, retinoid, crystallography

\section{INTRODUCTION}

Carotenoids are tetraterpene pigments naturally synthesized by plants and certain microorganisms, and are essential nutrients for animals (Walter and Strack, 2011; Harrison and Quadro, 2018). Their metabolic products participate in a variety of physiological processes such as retinoid signaling and vision cycle, and exert health-beneficial effects with antioxidant properties (von Lintig, 2010; Álvarez et al., 2014). Cleavage of carotenoids are catalyzed by carotenoid cleavage dioxygenases (CCDs) that share a seven-bladed $\beta$-propeller topology with a nonheme iron coordinated by four absolutely conserved histidines (Harrison and Bugg, 2014). The iron activates the dioxygen by a single electron transfer and generates the substrate radical intermediate, which then returns one electron to iron and forms the carbocation intermediate that ultimately yields aldehyde products (Sui et al., 2013; Guengerich and Yoshimoto, 2018).

The CCD family members are distributed from archaea to mammals and have diverse functions beyond carotenoid cleavage (Auldridge et al., 2006; Lobo et al., 2012). $\beta$-Carotene 15,15'-oxygenase (BCO1) (von Lintig and Vogt, 2000; Redmond et al., 2001) and $\beta$-carotene $9^{\prime}, 10^{\prime}$-oxygenase (BCO2) (Kiefer et al., 2001) are the two typical CCDs. Retinal pigment epithelium-specific $65 \mathrm{kDa}$ protein (RPE65), a CCD member, acts as a cis-trans retinoid isomerase of the polyene chain (Jin et al., 2005). Structural studies have been carried out on apocarotenoid-15,15'-oxygenase (ACO) from the cyanobacterium Synechocystis sp. PCC 6803 (Kloer et al., 2005), RPE65 from the ox Bos taurus (Kiser et al., 2009), 9-cis-epoxycarotenoid dioxygenase viviparous-14 (VP14) from the maize Zea mays (Messing et al., 2010), and CCD from the soil archaeon Nitrosotalea devanaterra (NdCCD) (Daruwalla et al., 2020). The diverse functions of CCDs are attributed to the unique structural variations in the helical dome that covers the active center in the $\beta$-propeller domain (Daruwalla and Kiser, 2020). However, no structure of BCO1 or BCO2 from any animals has been reported until 


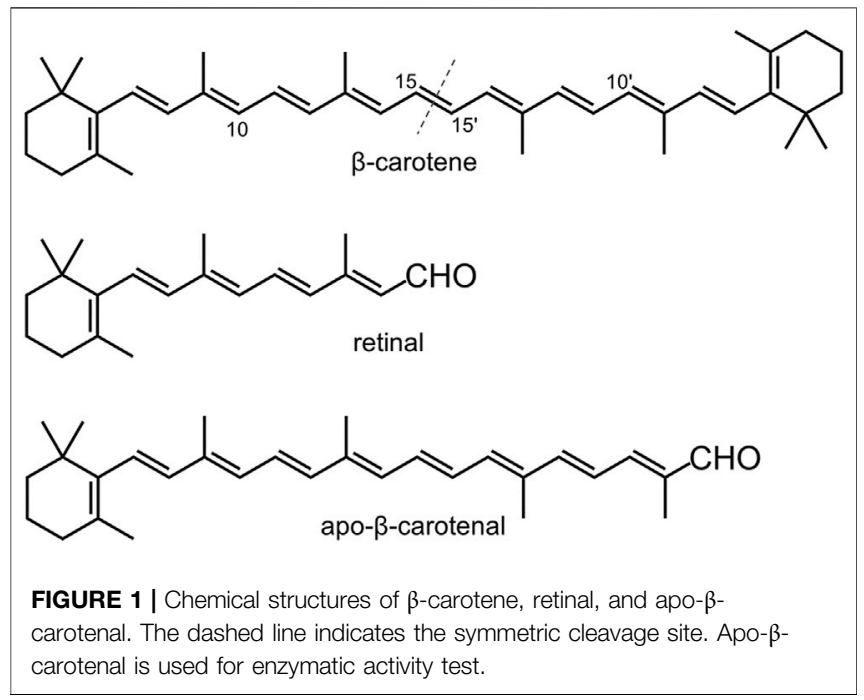

now, while their kinetics and substrate specificity have been investigated (Lindqvist and Andersson, 2002; dela Seña et al., 2013; Kelly et al., 2018; Bandara et al., 2021).

The genome of nematode Caenorhabditis elegans has two CCD genes, which are 17 centimorgan apart on chromosome II and are annotated as bcmo- 1 and bcmo-2 in WormBase. BCMO stands for $\beta$-carotene $15,15^{\prime}$-monooxygenase, although human BCO1 is actually a dioxygenase (dela Seña et al., 2014). As the name implies, the BCMO proteins are predicted to cleave $\beta$ carotene symmetrically (Figure 1). Here we use the annotated name in line with previous studies on $C$. elegans, in which BCMO-1 has been found to participate in cadmium-induced retinoic acid signaling (Cui et al., 2007; Cui and Freedman 2009). This work describes the crystal structures of BCMO-1 and BCMO-2. Compared with ACO, RPE65, VP14, and NdCCD, these two nematode proteins are most similar to the isomerase RPE65. In vitro activity assay did not detect their proposed ability for $\beta$-carotene cleavage, suggesting that they play other role than acting as $\mathrm{BCO}$ per se.

\section{MATERIALS AND METHODS}

\subsection{Protein Expression and Purification}

The C. elegans bcmo-1 and bcmo-2 genes (Y46G5A.24 and F53C3.12 in WormBase) were codon-optimized for expression in Escherichia coli, synthesized, and delivered in pUC57 by Sangon Biotech (Shanghai, China). Each gene was amplified by PCR and inserted between the restriction sites NdeI and XhoI of pET28a(+) (Novagen). The resulting two plasmids encode the 529-residue BCMO-1 and 530-residue BCMO-2, respectively, which are preceded by an N-terminal His6 tag. Each plasmid was transformed into E. coli BL21(DE3) cells (Novagen). The cells were grown at $37^{\circ} \mathrm{C}$ in $\mathrm{LB}$ medium containing $30 \mu \mathrm{g} / \mathrm{ml}$ kanamycin, and when the optical density at $600 \mathrm{~nm}$ reached $0.6 \sim 0.8$, expression was induced by $0.4 \mathrm{mM}$ isopropyl $\beta$-D-
TABLE 1 | Data collection and refinement statistics.

\begin{tabular}{|c|c|c|}
\hline Protein & ВСмо-1 & ВСМО-2 \\
\hline PDB code & 7WHO & 7WH1 \\
\hline \multicolumn{3}{|l|}{ Data collection } \\
\hline Space group & $\mathrm{P} 12_{1} 1$ & 14,22 \\
\hline Wavelength $(\AA)$ & 0.9792 & 0.9792 \\
\hline Resolution $(\AA)$ & $50-1.80(1.86-1.80)$ & $50-1.90(1.97-1.90)$ \\
\hline \multicolumn{3}{|l|}{ Unit cell } \\
\hline a, b, c (Å) & $73.2,104.7,76.4$ & $148.8,148.8,136.3$ \\
\hline$\alpha, \beta, \gamma\left(^{\circ}\right)$ & $90,102.2,90$ & $90,90,90$ \\
\hline Total reflections & 685107 (64934) & 1586788 (116653) \\
\hline Unique reflections & 102264 (9990) & 58459 (4402) \\
\hline Redundancy & $6.7(6.5)$ & $26.4(26.5)$ \\
\hline Completeness (\%) & $98.2(96.6)$ & $100(100)$ \\
\hline$|/ \sigma|$ & $29.1(3.0)$ & $41.7(2.3)$ \\
\hline$R_{\text {merge }}{ }^{\mathrm{a}}$ & $0.066(0.621)$ & $0.093(2.078)$ \\
\hline$R_{\text {pim }}{ }^{\mathrm{b}}$ & $0.027(0.259)$ & $0.018(0.409)$ \\
\hline$C C_{1 / 2}$ & $0.997(0.887)$ & $0.999(0.733)$ \\
\hline Wilson B-factor & 28.25 & 19.83 \\
\hline \multicolumn{3}{|l|}{ Refinement } \\
\hline Resolution (Å) & $31.63-1.80(1.86-1.80)$ & $39.49-1.90(1.97-1.90)$ \\
\hline$R_{\text {work }}{ }^{\mathrm{c} /} R_{\text {free }}{ }^{\mathrm{d}}$ & $0.166 / 0.189$ & $0.158 / 0.186$ \\
\hline No. of molecules & 2 & 1 \\
\hline No. of atoms & 9473 & 4638 \\
\hline Protein & 8546 & 4029 \\
\hline Ligand & 26 & 47 \\
\hline Water & 901 & 508 \\
\hline Average $B\left(\AA^{2}\right)$ & 35.32 & 27.35 \\
\hline Protein & 34.73 & 25.34 \\
\hline Ligand & 34.50 & 49.52 \\
\hline Water & 40.22 & 39.90 \\
\hline \multicolumn{3}{|l|}{ R.m.s deviations } \\
\hline Bond lengths ( $\AA$ ) & 0.007 & 0.007 \\
\hline Bond angles $\left({ }^{\circ}\right)$ & 0.89 & 0.84 \\
\hline \multicolumn{3}{|l|}{ Ramachandran plot } \\
\hline Favored (\%) & 96.58 & 96.54 \\
\hline Allowed (\%) & 3.42 & 3.46 \\
\hline
\end{tabular}

Values in parentheses are for highest resolution shell.

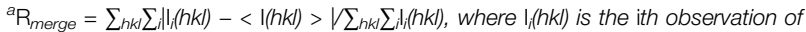
reflection $h \mathrm{kl}$ and $\langle\mid(h \mathrm{k})\rangle$ is the weighted intensity for all observations i of reflection $\mathrm{hkl}$. ${ }^{b} R_{\text {pim }}=R_{\text {merge }}[1 /(\mathrm{N}-1)]^{1 / 2}$.

${ }^{c} R_{\text {work }}=\sum|| F_{o}|-| F c|| \sum\left|F_{o}\right|$, where $F_{o}$ and $F_{c}$ are the observed and calculated structure factors, respectively.

${ }^{d} R_{\text {free }}$ is the cross-validated $R$ factor computed for a test set of $5 \%$ of the reflections, which were omitted during refinement.

thiogalactopyranoside. The cells were grown at $16^{\circ} \mathrm{C}$ for additional $18 \mathrm{~h}$ before harvested by centrifugation. The cell pellets were resuspended in lysis buffer $(0.3 \mathrm{M} \mathrm{NaCl}$ and $20 \mathrm{mM}$ Tris-Cl, $\mathrm{pH}$ 7.5) plus $20 \mathrm{mM}$ imidazole and lyzed by sonication at $0^{\circ} \mathrm{C}$. The lysate was cleared by centrifugation. The supernatant was loaded onto a nickel-chelating column (QIAGEN) and washed with the lysis buffer plus $20 \mathrm{mM}$ imidazole. The protein was eluted with $200 \mathrm{mM}$ imidazole in lysis buffer. The sample was concentrated by ultrafiltration for further purification by size exclusion chromatography (SEC) with a HiLoad 16/60 Superdex 200 column (GE Healthcare), which was equilibrated and eluted with the lysis buffer. The purity of the fractions was analyzed by SDS-PAGE. Expression and purification of mouse BCO1 was performed under the same condition. 


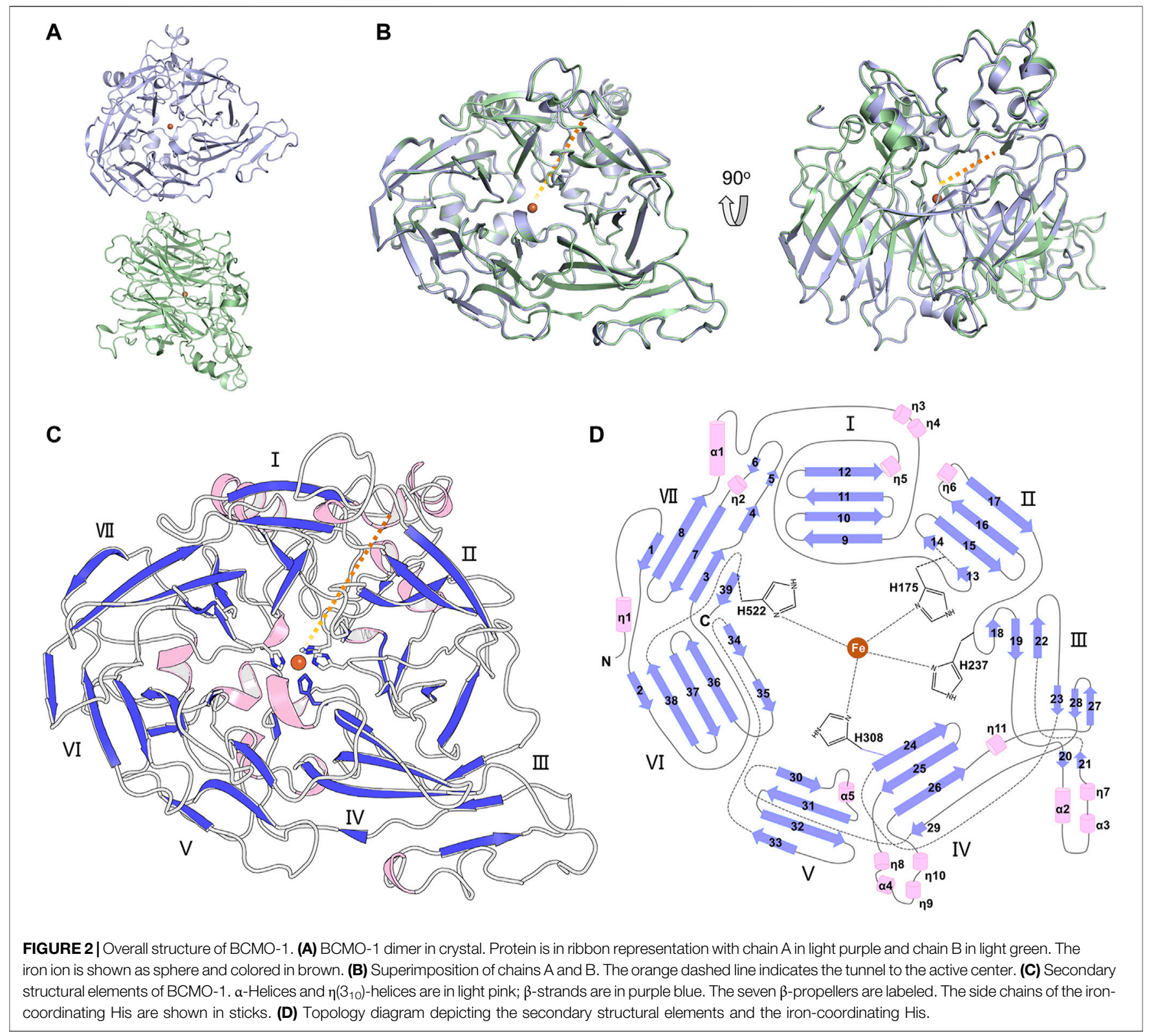

\subsection{Crystallization}

Crystal trays were set up at $16^{\circ} \mathrm{C}$ using the sitting-drop vapor diffusion method. Drops were prepared by mixing $1 \mu$ of purified protein and $1 \mu \mathrm{l}$ of reservoir solution against $150 \mu \mathrm{l}$ reservoir solution. The purified BCMO-1 was concentrated to $20 \mathrm{mg} / \mathrm{ml}$ in lysis buffer. Crystals were obtained using the reservoir solution of $0.2 \mathrm{M}$ ammonium sulfate, $0.1 \mathrm{M}$ Bis-Tris, $\mathrm{pH} 5.5$, and 25\% (w/v) polyethylene glycol 3,350. The purified BCMO-2 was concentrated to $36 \mathrm{mg} / \mathrm{ml}$ in a buffer containing $0.2 \mathrm{M} \mathrm{NaCl}$ and $20 \mathrm{mM}$ Tris-Cl, $\mathrm{pH} 7.5$, and crystals were obtained using the reservoir solution of $0.2 \mathrm{M}$ ammonium acetate and $2.2 \mathrm{M}$ ammonium sulfate. Before synchrotron data collection, the crystals were soaked in reservoir solution with $15 \%(\mathrm{v} / \mathrm{v})$ glycerol and flash-cooled in liquid nitrogen.

\subsection{Structure Determination and Structure Analysis}

X-ray diffraction data (Table 1) were recorded on beamlines BL18U1 and BL19U1 of the National Facility for Protein Sciences in Shanghai (Zhang et al., 2019). Data were processed using the HKL-3000 program package (Minor et al., 2006). The phase of BCMO-1 structure was solved by molecular replacement using Phaser (McCoy et al., 2007) in the CCP4 suite (Winn et al., 2011), with the coordinates of $B$. taurus RPE65 (PDB entry 4RSE) being used as search model (Kiser et al., 2015). Structural refinement was performed using Coot (Emsley and Cowtan, 2004) and PHENIX (Afonine et al., 2012). The structure of BCMO-2 was solved using the BCMO-1 structure as model. Model quality was evaluated by MolProbity (Chen et al., 2010). The atomic 

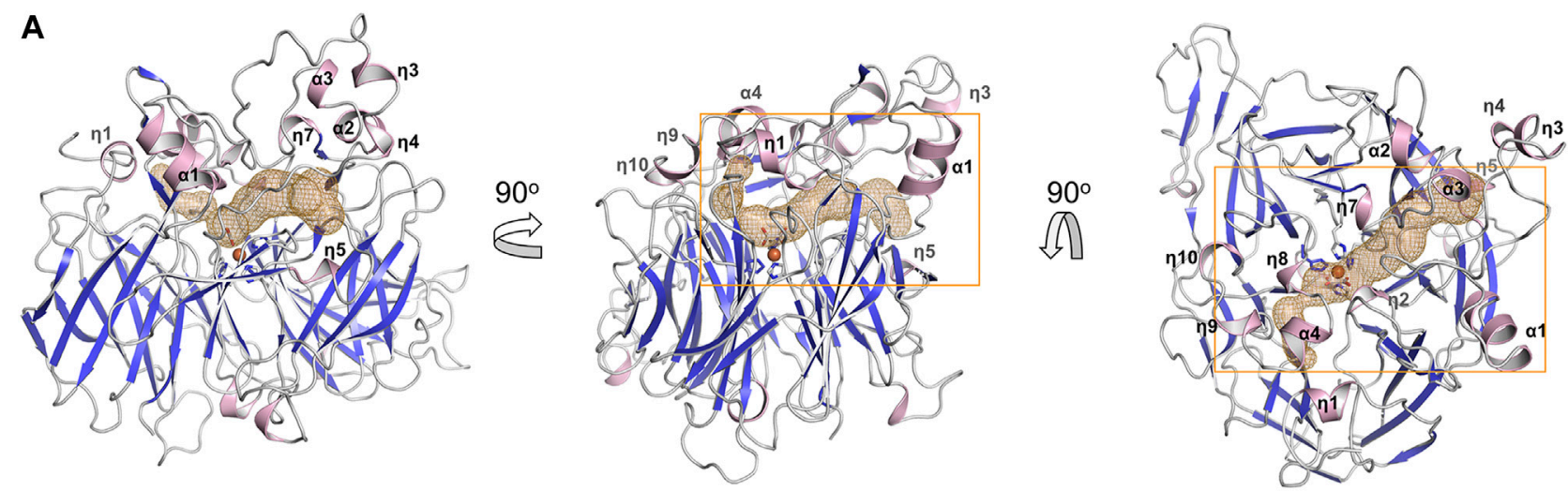

B
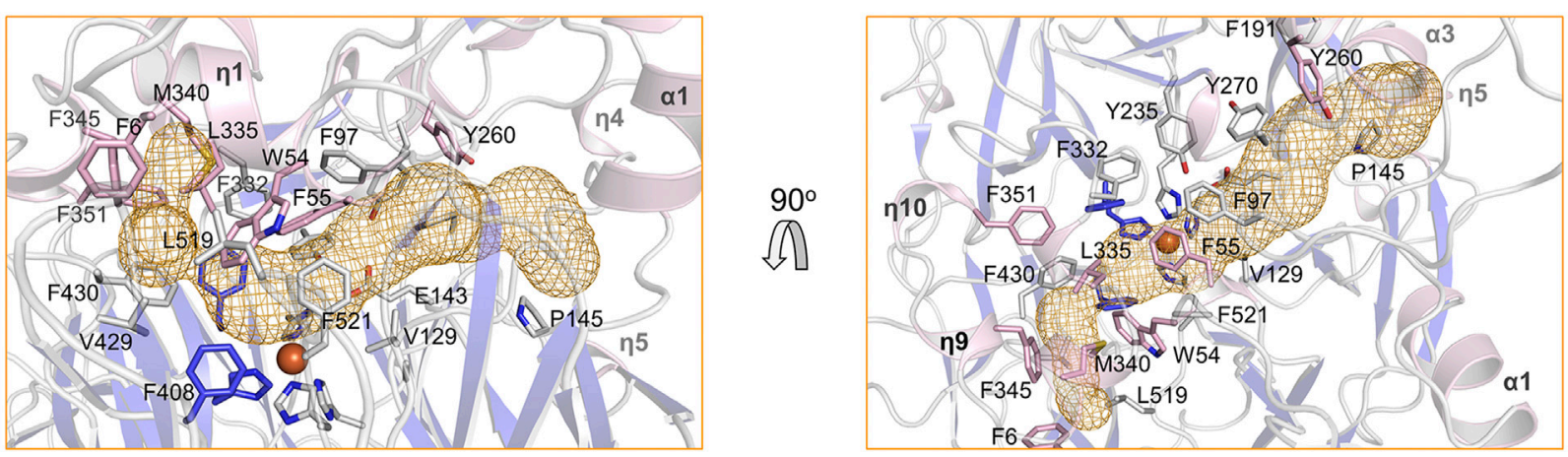

FIGURE 3 | Substrate tunnel of BCMO-1. (A) Ribbon representation of BCMO-1 and mesh representation of the substrate tunnel. The color scheme is as in Figure 1C; the tunnel is in orange; boxes denote zoomed regions shown in B. The orientation in the left panel is same to that in Figure 1B (right panel). (B) Zoomed-in view of the tunnel. Ribbons are in transparence; side chains of Phe6, Trp54, Phe55, Phe97, Val129, Glu143, Pro145, Phe191, Tyr235, Tyr260, Tyr270, Phe307, Phe332, Leu335, Met340, Phe345, Phe351, Phe408, Val429, Phe430, Leu519, Phe521, and the iron-coordinating His are shown as sticks.

coordinates and structure factors of BCMO-1 and BCMO-2 have been deposited in the Protein Data Bank under the accession codes 7WH0 and 7WH1, respectively. Tunnel analysis was performed with the toolkit MOLE (Pravda et al., 2018) and the result was imported into PyMOL (Schrödinger, LLC) for visualization. Alignment was performed with Clustal Omega (Madeira et al., 2019) and the output file was drawn using ESPript (Gouet et al., 2003).

\subsection{Enzymatic Activity Assay and HPLC Analysis}

The activity assay was performed based on established methods (Sui et al., 2015). Two typical CCD substrates, $\beta$-carotene and apo- $\beta$-carotenal (Figure 1), were used to test the enzymatic activity of BCMO-1 and BCMO-2. The positive control was mouse BCO1, which can cleave $\beta$-carotene and apo- $\beta$ carotenal and yield retinal (Redmond et al., 2001; Poliakov et al., 2005; Amengual et al., 2013). The recombinant mouse $\mathrm{BCO} 1$ protein was obtained in a similar manner to that used for BCMO- 1 and BCMO-2. The trans- $\beta$-carotene was obtained from Sigma-Aldrich; trans- $\beta$-apo- $8^{\prime}$-carotenal and retinal were purchased from Shanghai Aladdin Biochemical Tech; no further purification was performed. Assays were run at a final volume of $200 \mu \mathrm{l}$ consisting of $0.1 \mathrm{M}$ Tris- $\mathrm{HCl}, \mathrm{pH} 7.5,0.5 \mathrm{mM}$ dithiothreitol, $5 \%$ Tween $40,4 \mathrm{mM}$ sodium cholate, $15 \mathrm{mM}$ nicotinamide, and $18-\mu \mathrm{g}$ substrate $(\beta$-carotene or apo- $\beta$ carotenal), with $2.5 \mathrm{mM}$ (final) enzyme being added last to initiate the reaction. Reactions were kept in the dark at $37^{\circ} \mathrm{C}$ for $12 \mathrm{~h}$ before being quenched by addition of $50 \mu \mathrm{l}$ of $37 \%$ formaldehyde. Each quenched reaction was mixed with $250-\mu \mathrm{l}$ solvent A (methanol/water, 90/10, v/v) and 250- $\mu$ l solvent B (propan-2-ol/acetonitrile/water, 72/18/10, v/v/v), and was then filtered using a $0.22 \mu \mathrm{m}$ membrane to remove insoluble substrates. HPLC was performed using a Hypersil ODS C-18 column $(4.0 \times 250 \mathrm{~mm}$, Thermo Scientific $)$ on a Shimadzu $20 \mathrm{~A}$ system equipped with SPD-M40 UV-Vis detector. $40 \mu \mathrm{l}$ out of $\sim 750-\mu$ f filtered sample was injected into the column at a flow rate of $1 \mathrm{ml} / \mathrm{min}$ and at a column temperature of $28^{\circ} \mathrm{C}$. Separation was performed using the elution profile: $100 \%$ solvent A (0-5 min), $100 \%$ solvent B (5-30 min), and $100 \%$ solvent A (30-40 min). Separation was monitored at 380 and $448 \mathrm{~nm}$.

\section{RESULTS}

\subsection{BCMO-1 Structure}

The full-length BCMO-1 with an N-terminal His-tag was heterogenously expressed and purified by nickel affinity chromatography and then SEC. Purified BCMO-1 was 

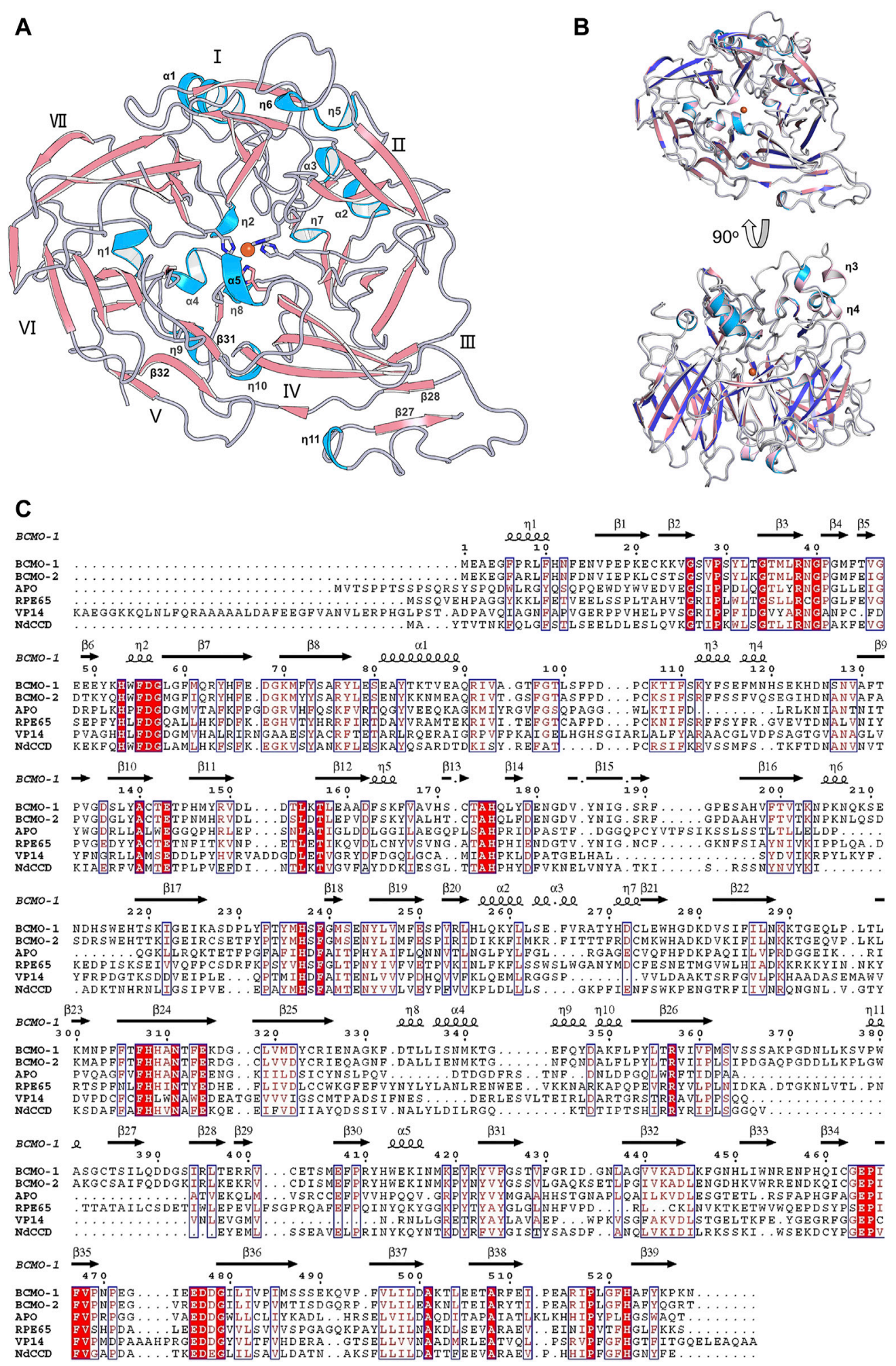

FIGURE 4 | Overall structure of BCMO-2 and comparison with BCMO-1. (A) Secondary structural elements of BCMO-2. $\alpha$-Helices and $\eta$-helices are in light blue and are labeled; $\beta$-strands are in light red. $\beta$-Strands $27,28,31$, and 32 are labeled. (B) Superimposition of BCMO- 1 and BCMO-2. BCMO-1 is colored as in Figure $2 \eta$ Helices 3 and 4 that are not observed in BCMO-2 are labeled. (C) Amino acid sequence alignment of BCMO-1, BCMO-2, ACO, RPE65, VP14, and NdCCD. The alignment figure was created by ESPript. Conserved residues are in red background, and similar residues are in red and boxed. Secondary structural elements of BCMO-1 are shown on the top of the alignment. 

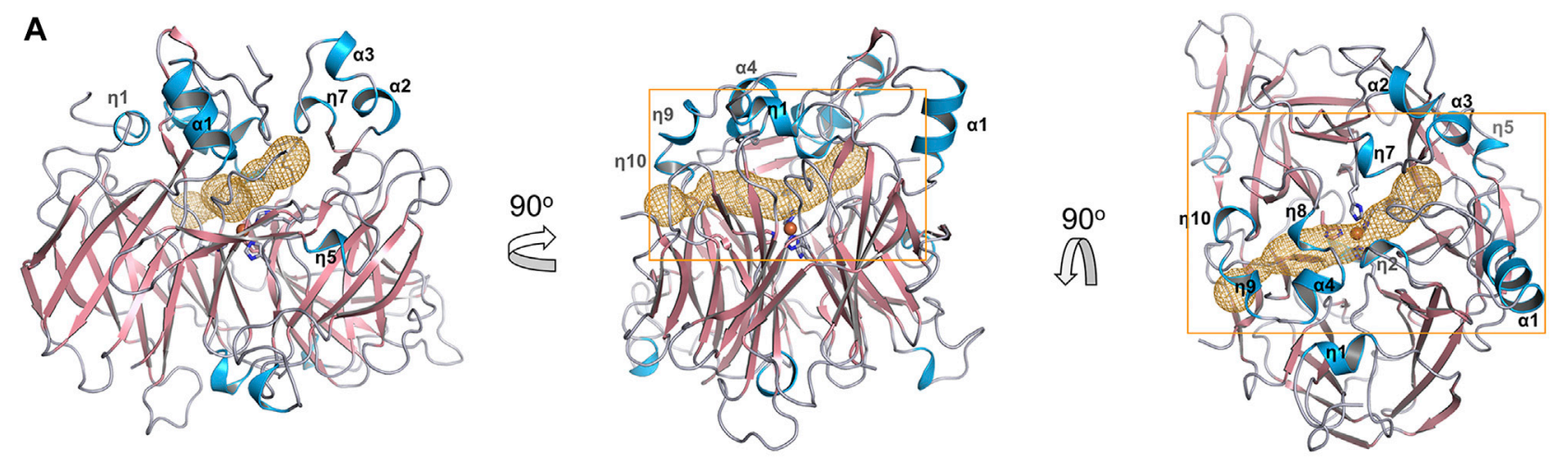

B
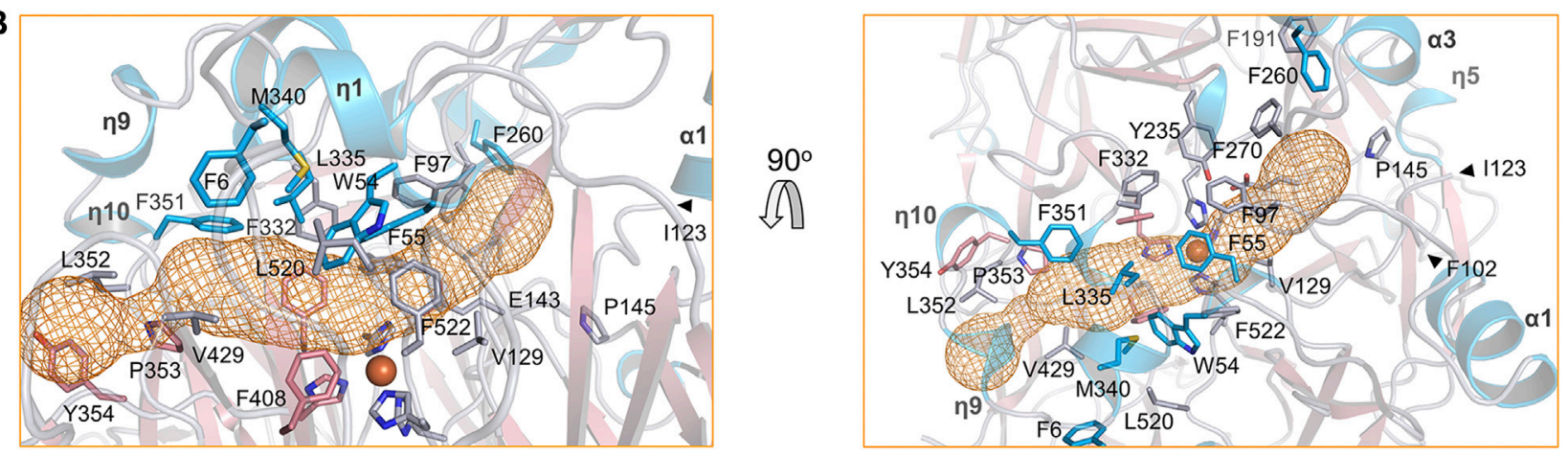

FIGURE 5 | Substrate tunnel of BCMO-2. (A) Ribbon representation of BCMO-2 and mesh representation of the tunnel. The color scheme is as in Figure 3. Box denotes the zoomed region shown in B. (B) Zoomed-in view of the tunnel. Ribbons are in transparence; side chains of Phe6, Trp54, Phe55, Phe97, Val129, Glu143, Pro145, Phe191, Tyr235, Phe260, Phe270, Phe307, Phe332, Leu335, Met340, Phe351-Tyr354, Phe408, Val429, Leu520, Phe522, and the iron-coordinating His are shown as sticks. The triangles denote Phe102 and lle123, the ends of traced backbone between domains VII and I.
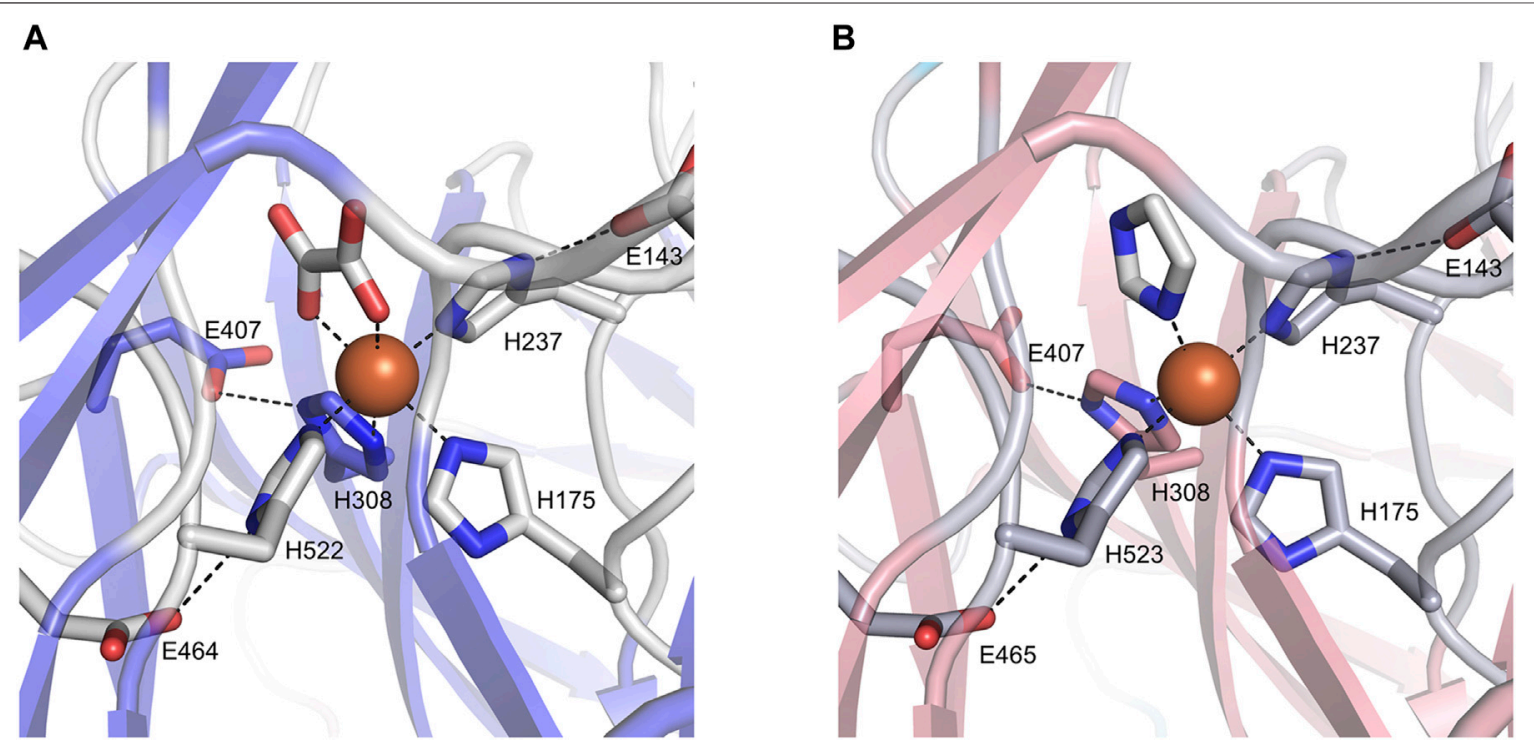

FIGURE 6 | The active centers. (A) BCMO-1 active center. Ribbons are in transparence; ligands and the side chains of residues constituting the iron center are shown as sticks; dashed lines denote metal coordination and the hydrogen bonds of Glu143, Glu407, and Glu464/465 with His237, His308 and His522/523, respectively. (B) BCMO-2 active center. 


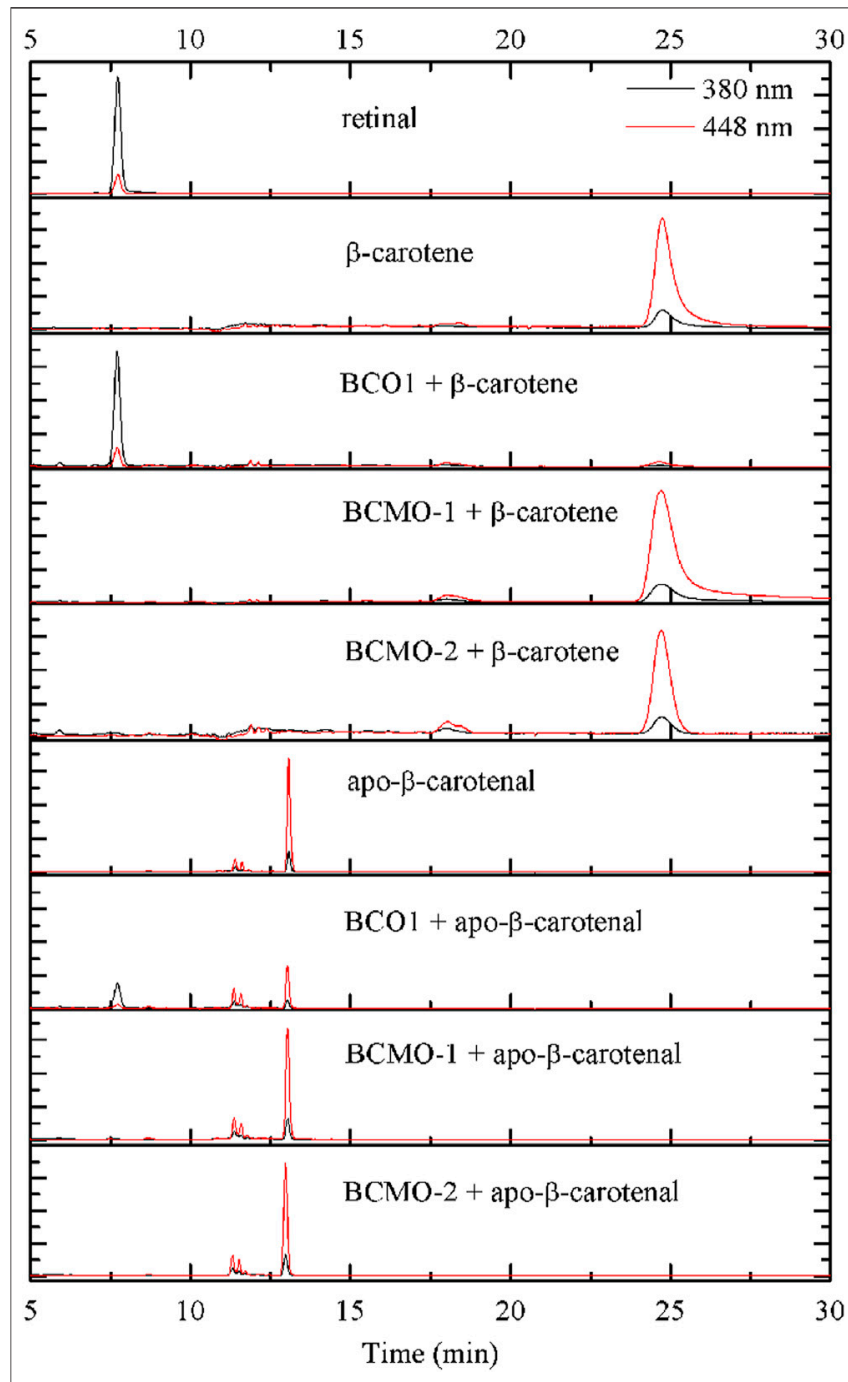

FIGURE 7 | BCMO- 1 and BCMO-2 do not cleave $\beta$-carotene or apo- $\beta$ carotenal. Chromatograms were recorded at $380 \mathrm{~nm}$ (black profile) and $448 \mathrm{~nm}$ (red profile). Separation of the substrate and product was achieved with propan-2-ol/acetonitrile/water (72/18/10, v/v/v) from time 5-30 min of HPLC analysis (described under Materials and Methods).

crystallized and the diffraction data were collected to $1.8-\AA$ resolution (Table 1). The structure was determined by molecular replacement, and two BCMO-1 molecules (chains A and $\mathrm{B}$ ) were found in an asymmetric unit (Figure 2A). The two chains are highly similar with a root-mean-square deviation (RMSD) of $0.20 \AA$, with the large differences occurring in the helical dome (Figure 2B) and reflecting the relative flexibility of these regions. A substrate access tunnel is located at the interface among the dome, blades I and II. Chain A is used for tertiary structure analysis and secondary structure definition (Figure 2C). BCMO- 1 has $39 \beta$-stands, $5 \alpha$-helices and $113_{10}$-helices as defined by the dictionary of secondary structure of proteins (DSSP) algorithm (Kabsch and Sander, 1983). The iron is coordinated by His175, His237, His308, and His522 from blades II, III, IV, and VII, respectively (Figure 2D).
The substrate tunnel is a bent cavity passing below the helical dome (Figure 3A). A narrow neck unequally divides the tunnel into two parts. The main part of tunnel (also indicated by the orange dashed line in Figure 2) runs from the entrance located between helices $\eta 4$ and $\eta 5$ to the iron center; the minor part heads to the interface between helices $\eta 1$ and $\alpha 4$, which is likely the exit passage of the product. Hydrophobicity of the tunnel is ensured by abundant lipophilic residues especially aromatic residues (Figure 3B). Trp54 and Phe 430 clamp the neck of the tunnel. The distance from the neck to the entrance along the main part of tunnel is approximate $30 \AA$, which is long enough to accommodate a carotene molecule.

\subsection{BCMO-2 Structure}

The full-length BCMO-2 was produced in same way as for BCMO-1, and was crystallized and diffracted to $1.9 \AA$ (Table 1). BCMO-2 has one molecule in an asymmetric unit and comprises $39 \beta$-stands, $5 \alpha$-helices and $933_{10}$-helices (Figure 4A). Compared with BCMO-1, two $33_{10}$-helices ( $\eta 3$ and $\eta 4$ in the loop connecting $\alpha 1$ and $\beta 9$ ) are not observed due to missing density for residues Pro103-Gly122 (Figure 4B). Residues Asp390-Ser392 from the $\beta 27-\beta 28$ loop and residues Leu430-Glu436 from the $\beta 31-\beta 32$ loop are not observed either. The $\beta 31-\beta 32$ loop hosts Ser435, the only insertion in BCMO-2 (530 residues in total) relative to BCMO-1 (529 residues). The boundaries of secondary structural elements are identical between BCMO-2 and BCMO-1 except that the numbering after residue Ser435 differs by one residue (Figure 4C). The RMSD value between BCMO-2 and BCMO- 1 (chain A) is $0.39 \AA$ for $429 \mathrm{Ca}$ atoms aligned, indicating that these two structures are highly similar. Therefore, consistent nomenclature of secondary structural elements is used for these two enzymes.

The substrate tunnel of BCMO-2 passes through the helical dome (Figure 5A). Its two ends are flanked by two unobserved fragments, Pro103-Gly122 and Leu430-Glu436, respectively. Helices $\eta 3$ and $\eta 4$ observed in BCMO- 1 are located within the first fragment, whose aromatic residues should provide a potential membrane-binding site adjacent to the tunnel. Because of missing electron density, the tunnel entrance that should be located between helices $\eta 4$ and $\eta 5$ shrinks inward (Figure 5B). The second missing fragment hosts Leu430, the counterpart of BCMO-1 Phe430, and its lack of electron density allows the tunnel runs towards $\eta 9$ and $\eta 10$. Despite differences at the two ends, the central part of the BCMO-2 tunnel is highly similar to that of BCMO-1, as revealed by the conserved hydrophobic residues.

\subsection{BCMO-1 and BCMO-2 Active Centers}

The ligand observed in the active center of BCMO- 1 was modeled as an oxalate. The dicarboxyl groups and imidazoles from His 175 , His237, His 308 , and His522 octahedrally coordinate the iron (Figure 6A). Three second-shell glutamates (Glu143, Glu407, and Glu464) stabilize His237, His308, and His522 via hydrogen bonds. The ligand bound to BCMO-2 was modeled as an imidazole, and the configuration of the BCMO-2 iron center was highly similar to that of BCMO-1 (Figure 6B). It should also be noted that the possibility of small ligand other than oxalate or 

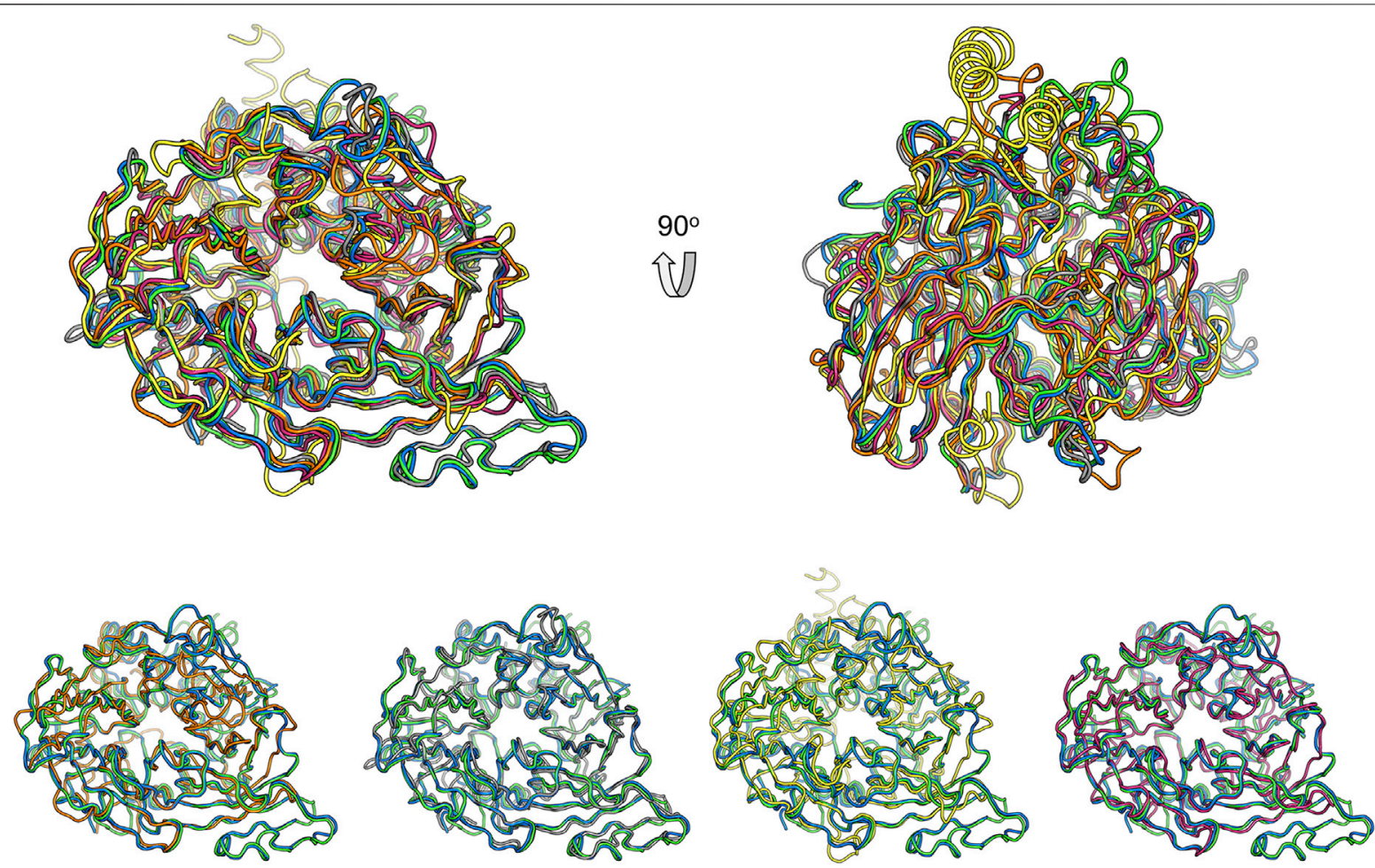

FIGURE 8 | Comparison of BCMO-1 and BCMO-2 with ACO, RPE65, VP14, and NdCCD. Superimposed structures are in tube representation. BCMO-1 and BCMO-2 are in green and blue, respectively. The coordinates of ACO (orange), RPE65 (gray), VP14 (yellow), and NdCCD (purple red) are from PDB entries 2BIW, 4RSE, 3NPE, and 6VCF, respectively. Superimposition of BCMO-1 and BCMO-2 with individual CCD protein is shown in the bottom panel.

imidazole cannot be excluded, nor the possibility that the ligands are responsible for the lack of detected activity.

\section{4 $\beta$-Carotene and Apo- $\beta$-Carotenal Cleavage Assay}

The activity of BCMO-1 and BCMO-2 was assayed with $\beta$-carotene and apo- $\beta$-carotenal using a 12 -h reaction time (Figure 7 ). Mouse BCO1 was used as positive control. Despite the annotation that BCMO-1 and BCMO-2 were $\beta$-carotene oxygenases, neither enzymes demonstrated $\beta$-carotene cleavage ability. When the asymmetric substrate apo- $\beta$-carotenal was tested, no cleaved product was detected either. These results were in contrast to those of mouse BCO1, which cleaved both substrates and produced retinal. The lack of evidence that BCMO-1 and BCMO2 are able to cleave $\beta$-carotene or apo- $\beta$-carotenal suggests that these two proteins are not functional orthologs of mammalian BCOs. It should be noted that the current assay conditions are suboptimal and the substrate scope still needs to be addressed.

\section{DISCUSSION}

Structural comparison with other CCD family members might provide clues for the function of these two nematode proteins. Superimposition of ACO (Kloer et al., 2005), RPE65 (Kiser et al., 2009), VP14 (Messing et al., 2010), and NdCCD (Daruwalla et al.,
2020) over BCMO-1 shows that their overall structures are similar (Figure 8). The RMSD values for the aligned $\mathrm{Ca}$ atoms between individual superimposed proteins (ACO, RPE65, VP14, and NdCCD) and BCMO-1/BCMO-2 are 1.56/1.71 $\AA$, 1.06/1.08 , $2.61 / 2.97 \AA$, and $1.15 / 1.17 \AA$, respectively, indicating that BCMO1 and BCMO-2 are more structurally similar to RPE65 and NdCCD than to ACO and VP14. The RMSD values are consistent with the amino-acid sequence identities between individual proteins and BCMO-1/BCMO-2, which are $25 \% / 26 \%, 34 \% / 33 \%$, $21 \% / 20 \%$, and $31 \% / 30 \%$, respectively. The major variations are located in the helical dome. In addition, BCMO-1, BCMO-2 and RPE65 share an insertion within blade III (including $\eta 11$ and $\beta 27$ in BCMO- 1 and BCMO-2), which is absent in ACO, VP14, and NdCCD. While the function of the two nematode CCDs still await further characterization, the structures reported here provide the first structural details for animal CCD members.

The physiological function of BCMO-1 has been characterized in C. elegans (Cui et al., 2007; Cui and Freedman, 2009), but the knowledge about BCMO-2 is scarce. The strikingly similarity between BCMO-1 and BCMO-2 (with an overall RMSD of $0.39 \AA$ for $429 \mathrm{Ca}$ atoms) highly suggests that they are functional homologs, as also reflected by their high sequence identity of $71 \%$. Such similarities suggest that they originate from a gene duplication event of the nematode line and are phylogenetically diverged from typical CCDs. BCMO-1 is involved in retinoic acid signaling and is expressed in C. elegans intestinal cells. Interestingly, intestinal cells are where retinal is detected (Wang et al., 2014). Considering the 
similarity to RPE65 and our unsuccessful attempt to detect their $\beta$ carotene cleavage ability, we propose that the two CCD members may work on some retinoid molecule. Although currently no difference is observed when retinal is tested, this possibility awaits to be addressed in future studies.

In summary, we have solved the structures of BCMO- 1 and BCMO-2 that belong to the CCD family. A structural comparison with the known structures of CCDs suggests a role other than $\beta$ carotene cleavage, which is consistent with in vitro functional data. These two worm CCD members are likely to be functionally similar to the retinoid isomerase.

\section{DATA AVAILABILITY STATEMENT}

The datasets presented in this study can be found in online repositories. The names of the repository/repositories and accession number(s) can be found below: https://www.wwpdb. org/pdb?id=pdb_00007wh0; https://www.wwpdb.org/pdb?id=pdb_ 00007wh1.

\section{REFERENCES}

Afonine, P. V., Grosse-Kunstleve, R. W., Echols, N., Headd, J. J., Moriarty, N. W., Mustyakimov, M., et al. (2012). Towards Automated Crystallographic Structure Refinement With phenix.Refine. Acta Crystallogr. D Biol. Cryst. 68, 352-367. doi:10.1107/s0907444912001308

Álvarez, R., Vaz, B., Gronemeyer, H., and de Lera, Á. R. (2014). Functions, Therapeutic Applications, and Synthesis of Retinoids and Carotenoids. Chem. Rev. 114, 1-125. doi:10.1021/cr400126u

Amengual, J., Widjaja-Adhi, M. A. K., Rodriguez-Santiago, S., Hessel, S., Golczak, M., Palczewski, K., et al. (2013). Two Carotenoid Oxygenases Contribute to Mammalian Provitamin A Metabolism. J. Biol. Chem. 288, 34081-34096. doi:10.1074/jbc.m113.501049

Auldridge, M. E., McCarty, D. R., and Klee, H. J. (2006). Plant Carotenoid Cleavage Oxygenases and Their Apocarotenoid Products. Curr. Opin. Plant Biol. 9, 315-321. doi:10.1016/j.pbi.2006.03.005

Bandara, S., Thomas, L. D., Ramkumar, S., Khadka, N., Kiser, P. D., Golczak, M., et al. (2021). The Structural and Biochemical Basis of Apocarotenoid Processing by $\beta$-Carotene Oxygenase-2. ACS Chem. Biol. 16, 480-490. doi:10.1021/ acschembio.0c00832

Chen, V. B., Arendall, W. B., 3rd, Headd, J. J., Keedy, D. A., Immormino, R. M., Kapral, G. J., et al. (2010). MolProbity: All-Atom Structure Validation for Macromolecular Crystallography. Acta Crystallogr. D Biol. Cryst. 66, 12-21. doi:10.1107/s0907444909042073

Cui, Y., and Freedman, J. H. (2009). Cadmium Induces Retinoic Acid Signaling by Regulating Retinoic Acid Metabolic Gene Expression. J. Biol. Chem. 284, 24925-24932. doi:10.1074/jbc.m109.026609

Cui, Y., McBride, S. J., Boyd, W. A., Alper, S., and Freedman, J. H. (2007). Toxicogenomic Analysis of Caenorhabditis elegans Reveals Novel Genes and Pathways Involved in the Resistance to Cadmium Toxicity. Genome Biol. 8, R122. doi:10.1186/gb-2007-8-6-r122

Daruwalla, A., and Kiser, P. D. (2020). Structural and Mechanistic Aspects of Carotenoid Cleavage Dioxygenases (CCDs). Biochim. Biophys. Acta (Bba) Mol. Cell Biol. Lipids. 1865, 158590. doi:10.1016/j.bbalip.2019.158590

Daruwalla, A., Zhang, J., Lee, H. J., Khadka, N., Farquhar, E. R., Shi, W., et al. (2020). Structural Basis for Carotenoid Cleavage by an Archaeal Carotenoid Dioxygenase. Proc. Natl. Acad. Sci. USA. 117, 19914-19925. doi:10.1073/pnas. 2004116117

dela Seña, C., Narayanasamy, S., Riedl, K. M., Curley, R. W., Jr., Schwartz, S. J., and Harrison, E. H. (2013). Substrate Specificity of Purified Recombinant Human $\beta$ -

\section{AUTHOR CONTRIBUTIONS}

WP, Y-LZ, and JW performed the experiments; WP, H-ED, and $\mathrm{XW}$ analyzed the structure; LL designed the study; H-ED, XW, and LL wrote the paper.

\section{FUNDING}

This work was supported by the National Natural Science Foundation of China (grant 31870729) and an intramural start-up fund from Anhui University.

\section{ACKNOWLEDGMENTS}

We thank Mingzhu Wang from Anhui University and the staffs from BL18U1 and BL19U1 beamlines of National Facility for Protein Science in Shanghai (NFPSS) at Shanghai Synchrotron Radiation Facility for assistance during data collection.

Carotene 15,15'-Oxygenase (BCO1). J. Biol. Chem. 288, 37094-37103. doi:10. 1074/jbc.M113.507160

dela Seña, C., Riedl, K. M., Narayanasamy, S., Curley, R. W., Jr., Schwartz, S. J., and Harrison, E. H. (2014). The Human Enzyme that Converts Dietary Provitamin A Carotenoids to Vitamin A Is a Dioxygenase. J. Biol. Chem. 289, 13661-13666. doi:10.1074/jbc.M114.557710

Emsley, P., and Cowtan, K. (2004). Coot: Model-Building Tools for Molecular Graphics. Acta Crystallogr. D Biol. Cryst. 60, 2126-2132. doi:10.1107/ s0907444904019158

Gouet, P., Robert, X., and Courcelle, E. (2003). ESPript/ENDscript: Extracting and Rendering Sequence and 3D Information from Atomic Structures of Proteins. Nucleic Acids Res. 31, 3320-3323. doi:10.1093/nar/gkg556

Guengerich, F. P., and Yoshimoto, F. K. (2018). Formation and Cleavage of C-C Bonds by Enzymatic Oxidation-Reduction Reactions. Chem. Rev. 118, 6573-6655. doi:10.1021/acs.chemrev.8b00031

Harrison, E. H., and Quadro, L. (2018). Apocarotenoids: Emerging Roles in Mammals. Annu. Rev. Nutr. 38, 153-172. doi:10.1146/annurev-nutr-082117051841

Harrison, P. J., and Bugg, T. D. H. (2014). Enzymology of the Carotenoid Cleavage Dioxygenases: Reaction Mechanisms, Inhibition and Biochemical Roles. Arch. Biochem. Biophys. 544, 105-111. doi:10.1016/j.abb.2013.10.005

Jin, M., Li, S., Moghrabi, W. N., Sun, H., and Travis, G. H. (2005). Rpe65 Is the Retinoid Isomerase in Bovine Retinal Pigment Epithelium. Cell. 122, 449-459. doi:10.1016/j.cell.2005.06.042

Kabsch, W., and Sander, C. (1983). Dictionary of Protein Secondary Structure: Pattern Recognition of Hydrogen-Bonded and Geometrical Features. Biopolymers. 22, 2577-2637. doi:10.1002/bip.360221211

Kelly, M. E., Ramkumar, S., Sun, W., Colon Ortiz, C., Kiser, P. D., Golczak, M., et al. (2018). The Biochemical Basis of Vitamin A Production from the Asymmetric Carotenoid $\beta$-Cryptoxanthin. ACS Chem. Biol. 13, 2121-2129. doi:10.1021/ acschembio. 8 b00290

Kiefer, C., Hessel, S., Lampert, J. M., Vogt, K., Lederer, M. O., Breithaupt, D. E., et al. (2001). Identification and Characterization of a Mammalian Enzyme Catalyzing the Asymmetric Oxidative Cleavage of Provitamin A. J. Biol. Chem. 276, 14110-14116. doi:10.1074/jbc.m011510200

Kiser, P. D., Golczak, M., Lodowski, D. T., Chance, M. R., and Palczewski, K. (2009). Crystal Structure of Native RPE65, the Retinoid Isomerase of the Visual Cycle. Proc. Natl. Acad. Sci. 106, 17325-17330. doi:10.1073/pnas.0906600106

Kiser, P. D., Zhang, J., Badiee, M., Li, Q., Shi, W., Sui, X., et al. (2015). Catalytic Mechanism of a Retinoid Isomerase Essential for Vertebrate Vision. Nat. Chem. Biol. 11, 409-415. doi:10.1038/nchembio.1799 
Kloer, D. P., Ruch, S., Al-Babili, S., Beyer, P., and Schulz, G. E. (2005). The Structure of a Retinal-Forming Carotenoid Oxygenase. Science. 308, 267-269. doi:10. 1126/science. 1108965

Lindqvist, A., and Andersson, S. (2002). Biochemical Properties of Purified Recombinant Human $\beta$-Carotene 15,15'-Monooxygenase. J. Biol. Chem. 277, 23942-23948. doi:10.1074/jbc.m202756200

Lobo, G. P., Amengual, J., Palczewski, G., Babino, D., and von Lintig, J. (2012). Mammalian Carotenoid-Oxygenases: Key Players for Carotenoid Function and Homeostasis. Biochim. Biophys. Acta (Bba) - Mol. Cell Biol. Lipids. 1821, 78-87. doi:10.1016/j.bbalip.2011.04.010

Madeira, F., Park, Y. m., Lee, J., Buso, N., Gur, T., Madhusoodanan, N., et al. (2019). The EMBL-EBI Search and Sequence Analysis Tools APIs in 2019. Nucleic Acids Res. 47, W636-W641. doi:10.1093/nar/gkz268

McCoy, A. J., Grosse-Kunstleve, R. W., Adams, P. D., Winn, M. D., Storoni, L. C., and Read, R. J. (2007). Phasercrystallographic Software. J. Appl. Cryst. 40, 658-674. doi:10.1107/s0021889807021206

Messing, S. A. J., Gabelli, S. B., Echeverria, I., Vogel, J. T., Guan, J. C., Tan, B. C., et al. (2010). Structural Insights into maize Viviparous14, a Key Enzyme in the Biosynthesis of the Phytohormone Abscisic Acid. Plant Cell. 22, 2970-2980. doi:10.1105/tpc.110.074815

Minor, W., Cymborowski, M., Otwinowski, Z., and Chruszcz, M. (2006). HKL3000: the Integration of Data Reduction and Structure Solution - from Diffraction Images to an Initial Model in Minutes. Acta Crystallogr. D Biol. Cryst. 62, 859-866. doi:10.1107/s0907444906019949

Poliakov, E., Gentleman, S., Cunningham, F. X., Jr, Miller-Ihli, N. J., and Redmond, T. M. (2005). Key Role of Conserved Histidines in Recombinant Mouse $\beta$ Carotene 15,15'-Monooxygenase-1 Activity. J. Biol. Chem. 280, 29217-29223. doi:10.1074/jbc.m500409200

Pravda, L., Sehnal, D., Toušek, D., Navrátilová, V., Bazgier, V., Berka, K., et al. (2018). MOLEonline: a Web-Based Tool for Analyzing Channels, Tunnels and Pores (2018 Update). Nucleic Acids Res. 46, W368-W373. doi:10.1093/nar/ gky309

Redmond, T. M., Gentleman, S., Duncan, T., Yu, S., Wiggert, B., Gantt, E., et al. (2001). Identification, Expression, and Substrate Specificity of a Mammalian $\beta$ Carotene 15,15'-Dioxygenase. J. Biol. Chem. 276, 6560-6565. doi:10.1074/jbc. $\mathrm{m} 009030200$

Sui, X., Golczak, M., Zhang, J., Kleinberg, K. A., von Lintig, J., Palczewski, K., et al. (2015). Utilization of Dioxygen by Carotenoid Cleavage Oxygenases. J. Biol. Chem. 290, 30212-30223. doi:10.1074/jbc.m115.696799
Sui, X., Kiser, P. D., Lintig, J. v., and Palczewski, K. (2013). Structural Basis of Carotenoid Cleavage: from Bacteria to Mammals. Arch. Biochem. Biophys. 539, 203-213. doi:10.1016/j.abb.2013.06.012

von Lintig, J. (2010). Colors with Functions: Elucidating the Biochemical and Molecular Basis of Carotenoid Metabolism. Annu. Rev. Nutr. 30, 35-56. doi:10. 1146/annurev-nutr-080508-141027

von Lintig, J., and Vogt, K. (2000). Filling the Gap in Vitamin A Research. J. Biol. Chem. 275, 11915-11920. doi:10.1074/jbc.275.16.11915

Walter, M. H., and Strack, D. (2011). Carotenoids and Their Cleavage Products: Biosynthesis and Functions. Nat. Prod. Rep. 28, 663-692. doi:10.1039/c0np00036a

Wang, P., Liu, B., Zhang, D., Belew, M. Y., Tissenbaum, H. A., and Cheng, J.-X. (2014). Imaging Lipid Metabolism in LiveCaenorhabditis elegansUsing Fingerprint Vibrations. Angew. Chem. Int. Ed. 53, 11787-11792. doi:10. 1002/anie.201406029

Winn, M. D., Ballard, C. C., Cowtan, K. D., Dodson, E. J., Emsley, P., Evans, P. R., et al. (2011). Overview of theCCP4 Suite and Current Developments. Acta Crystallogr. D Biol. Cryst. 67, 235-242. doi:10.1107/s0907444910045749

Zhang, W.-Z., Tang, J.-C., Wang, S.-S., Wang, Z.-J., Qin, W.-M., and He, J.-H. (2019). The Protein Complex Crystallography Beamline (BL19U1) at the Shanghai Synchrotron Radiation Facility. Nucl. Sci. Tech. 30, 170. doi:10. 1007/s41365-019-0683-2

Conflict of Interest: The authors declare that the research was conducted in the absence of any commercial or financial relationships that could be construed as a potential conflict of interest.

Publisher's Note: All claims expressed in this article are solely those of the authors and do not necessarily represent those of their affiliated organizations, or those of the publisher, the editors and the reviewers. Any product that may be evaluated in this article, or claim that may be made by its manufacturer, is not guaranteed or endorsed by the publisher.

Copyright (C) 2022 Pan, Zhou, Wang, Dai, Wang and Liu. This is an open-access article distributed under the terms of the Creative Commons Attribution License (CC $B Y)$. The use, distribution or reproduction in other forums is permitted, provided the original author(s) and the copyright owner(s) are credited and that the original publication in this journal is cited, in accordance with accepted academic practice. No use, distribution or reproduction is permitted which does not comply with these terms. 